\title{
REVISÃO NARRATIVA SOBRE SCHADENFREUDE: O PRAZER MALICIOSO E AS RELAÇÕES INTERGRUPAIS
}

Narrative Review on Schadenfreude: Malicious Pleasure and Intergroup Relations

Revisión Narrativa sobre Schadenfreude: El Placer Malicioso y las Relaciones Entre Grupos

Revue narrative sur Schadenfreude : Le Plaisir Malveillant et les Relations Intergroupes

doi) $10.5020 / 23590777 . r s . v 20 i E s p 1 . e 8817$

\section{Saulo Santos Menezes Almeida 9 iD}

Doutor em Psicologia pela Universidade Federal da Bahia. Coordenador e Professor Adjunto do curso de Psicologia da Universidade Salvador.

\section{Vanessa Andrade de Assis}

Graduanda em Psicologia pela Universidade Salvador (UNIFACS). Pós Graduanda em Neuropsicologia pelo Núcleo de Pós Graduação Gastão Guimarães

\section{Ícaro Batista de Cerqueira 9}

Docente no Curso de Psicologia da Faculdade Pitágoras. Especialista em Saúde Mental com ênfase em dependência química (CESDA), Pós Graduando em Terapia Cognitivo Comportamental (UNISEPE).

\section{Resumo}

Schadenfreude é um termo alemão que tem como significado o prazer malicioso frente à adversidade alheia. Esse sentimento possui relação com inúmeras variáveis, entre as quais: a inveja, o merecimento e os relacionamentos intergrupais. Sendo assim, esse sentimento pode impactar o contato entre os indivíduos, tanto de forma positiva quanto negativa. A importância do referido assunto para as relações sociais, econômicas e políticas, bem como as contradições e interrogações da schadenfreude, com a escassez de pesquisas dentro do âmbito nacional, fomentaram a construção desta revisão narrativa. A partir da literatura encontrada, foram contemplados 17 artigos, nacionais e internacionais, utilizando como critério de inclusão o termo schadenfreude no título ou nas palavras-chave. Além disso, deveriam relacionarse à área da Psicologia, enfatizando seus resultados e suas limitações para extensão do conhecimento sobre o tema e para demarcações em direção à construção de novas pesquisas nessa área. Dessa forma, este artigo possibilita um entendimento da perspectiva e da compreensão do panorama sobre os estudos da schadenfreude, sendo associado com termos como simpatia, merecimento do infortúnio, moralidade e mecanismo evolutivo de aversão à iniquidade. Observa-se que a produção científica nacional ainda é escassa, de modo que se faz necessário seu crescimento, assim como das produções internacionais. Um fator positivo é a existência de estudos com levantamentos empíricos de coleta de dados que encontraram resultados efetivos, demonstrando que essa é uma área que pode ser mais bem explorada.

Palavras-chave: schadenfreude; relações grupais; revisão narrativa.

\section{Abstract}

Schadenfreude is a German term that means malicious pleasure in the face of adversity. This feeling is related to innumerable variables, among which: envy, deserving, and intergroup relationships. Therefore, this feeling can impact the contact between individuals, both positively and negatively. The importance of this subject for social, economic, and political relations, as well as the contradictions and questions of schadenfreude, with the scarcity of research within the national scope, fostered the construction of this narrative review. From the literature found, 17 national and international articles were considered, using the term schadenfreude in the title or keywords as inclusion criteria. Besides, they should relate to the area of Psychology, emphasizing its results and its limitations for the extension of knowledge on the subject and demarcations towards the construction of new research in this area Thus, this article provides an understanding of the 
perspective and understanding of the panorama on schadenfreude studies, being associated with terms such as sympathy, deserving of misfortune, morality and the evolutionary mechanism of aversion to iniquity. It is observed that the national scientific production is still scarce, so its growth is necessary, as well as international productions. A positive factor is the existence of studies with empirical surveys of data collection that found effective results, demonstrating that this is an area that can be better explored.

Keywords: schadenfreude; group relations; narrative review.

\section{Resumen}

Schadenfreude es un término malicioso alemán que significa placer malicioso ante la adversidad ajena. Este sentimiento posee relación con inúmeras variables, entre las cuales: la envidia, el merecimiento y los relacionamientos entre grupos. De esta forma, este sentimiento puede impactar el contacto entre los individuos, tanto de forma positiva cuanto de forma negativa. La importancia del referido tema para las relaciones sociales, económicas y políticas, como también las contradicciones y cuestionamientos de la schadenfreude, con la escasez de investigaciones dentro del ámbito nacional, fomentaron la construcción de esta revisión narrativa. A partir de la literatura encontrada, fueron seleccionados 17 artículos, nacionales e internacionales, utilizando como criterio de inclusión el término schadenfreude en el título o en las palabras-clave. Además, deberían relacionarse a la Psicología, con énfasis en sus resultados y sus limitaciones para extensión del conocimiento sobre el tema y para demarcación en dirección a la construcción de nuevas investigaciones en esta área. De este modo, este trabajo posibilita una comprensión de la perspectiva y del panorama sobre los estudios de la schadenfreude, asociando con términos como simpatía, merecimiento de la dificultad, moralidad y mecanismo evolutivo de aversión a la iniquidad. Se observa que la producción científica nacional todavía es escasa, de modo que se hace necesario su crecimiento, como también de las producciones internacionales. Un factor positivo es la existencia de trabajos con recopilación empírica de colecta de datos que encontrarán resultados efectivos, demostrando que esta es una área que puede ser mejor explorada.

Palabras clave: schadenfreude; relaciones grupales; revisión narrativa.

\section{Résumé}

«Schadenfreude» est un terme allemand qui signifie plaisir malveillant face à l'adversité de l'autre. Ce sentiment est lié à d'innombrables variables, parmi lesquelles : l'envie, le mérite et les relations intergroupes. Par conséquent, ce sentiment peut avoir un impact positif et négatif sur le contact entre les individus. L'importance de ce sujet pour les relations sociales, économiques et politiques, ainsi que les contradictions et les questions de «schadenfreude», avec la rareté des recherches à l'échelle nationale, ont favorisé la construction de cette revue narrative. À partir de la littérature trouvée, 17 articles, nationaux et internationaux, ont été considérés, en utilisant le terme «schadenfreude» dans le titre ou dans les mots clés comme critères d'inclusion. En outre, ils doivent concerner le domaine de la Psychologie, en mettant en relief leurs résultats et leurs limites pour l'extension des connaissances sur le sujet et pour les démarcations vers la construction de nouvelles recherches dans ce domaine. Ainsi, cet article rend possible comprendre la perspective et la compréhension du panorama sur les études de «schadenfreude», lequel est associé à des termes tels que sympathie, méritant le malheur, moralité et mécanisme évolutif d'aversion à l'iniquité. On constate que la production scientifique nationale (bien comme les productions internationales) sur ce thème est encore rare, de sorte que sa croissance est nécessaire. Un facteur positif est l'existence d'études avec des enquêtes empiriques de collecte de données qui ont trouvé des résultats efficaces. Cela démontre un domaine qui peut être mieux exploré.

Mots-clés : schadenfreude; relations de groupe; revue narrative.

Schadenfreude é um termo alemão que significa prazer malicioso, ou seja, é quando o ser humano sente prazer pela infelicidade do outro. Esse prazer não acontece por propiciar o sofrimento ao outro, mas, sim, por observar esse infortúnio. Algumas variáveis, como a inveja (desejo em possuir um bem ou característica de outrem) e o merecimento (crença de que determinado indivíduo ou grupo possui mérito para alcançar determinados bens ou reconhecimentos sociais), além dos limites entre o endo/exogrupo (o sentimento de pertencimento a um grupo e o que separa o meu grupo dos outros em termos de propriedades física, intelectuais, emocionais ou mesmo sociais), são algumas das variáveis que se relacionam com esse fenômeno. No campo da inveja, por exemplo, tem sido constatado que os alvos superiores/invejados são os mais vulneráveis para a expressão da schadenfreude, porém a proximidade que o observador tem do alvo de infortúnio também poderá influenciar (Van de Ven et al., 2014).

Na relação entre schadenfreude e as relações intergrupais, salienta-se que emoções advêm do resultado de episódios e interações que visam o bem-estar do endogrupo, independente do envolvimento do indivíduo com o evento. Para o grupo 
adquirir um significado afetivo e emocional no indivíduo, é preciso que ele se identifique com este, pois só assim se sentirá membro integrante do grupo, o qual irá se tornar uma parte do self do indivíduo. Esse forte sentimento de identificação com o grupo possibilitará um maior prazer quando um evento de infelicidade beneficiar o exogrupo, mesmo que o acontecimento possa ser prejudicial para inocentes. Dessa forma, a schadenfreude se situa como distância psicológica e divergência emocional, pois cita o prazer como uma resposta divergente ao sofrimento do outro (Spurgin, 2015).

Assim sendo, a expressão de schadenfreude traz implicações tanto positivas quanto negativas nas relações intergrupais, sendo um importante meio para a compreensão da complexidade da vida social, econômica, política e cultural. Ademais, é um tema ainda pouco estudado no Brasil, inclusive pela área da Psicologia, e ainda imerso por inúmeras contradições e interrogações, sendo imprescindível a realização de pesquisas na área para amplificar o conhecimento sobre esse tipo de prazer malicioso.

Como exemplo dessas interrogações, Combs, Powell, Sxhurtz, e Smith (2009) apontam a possibilidade de a maioria das experiências de schadenfreude serem exemplos de sentimentos ambivalentes ou mistos, já que é improvável que pessoas que são bem socializadas se sintam totalmente à vontade com o sofrimento de outras pessoas, especialmente quando se sentem egoístas nessa expressão, além de ser difícil ter a certeza de quantos correlatos de schadenfreude são sentimentos reais dos participantes, pois os relatos podem ser autocensurados, mesmo inconscientemente.

Assim, compreender suas consequências trará benefícios para o indivíduo e para a sociedade em geral, à medida que se solidifica como uma importante ferramenta para a assimilação das trocas sociais. Portanto, o objetivo deste artigo é a realização de uma revisão narrativa sobre a schadenfreude, apresentando diversos estudos realizados nos últimos anos, enfatizando seus resultados e suas limitações para amplificação do conhecimento sobre o tema na área da Psicologia e para delineamento de construção de novas pesquisas na área.

\section{Método}

Para este trabalho, utiliza-se a revisão narrativa, que é apropriada para descrever e discutir o desenvolvimento de um determinado assunto do ponto de vista teórico ou contextual. A revisão narrativa constitui-se, basicamente, de análise da literatura publicada em livros e artigos de revistas impressas e/ou eletrônicas, e na interpretação e análise crítica pessoal do autor. Portanto, este artigo pretende exercer fundamentos para a aquisição e atualização do conhecimento sobre a schadenfreude (Rother, 2007).

A revisão narrativa de literatura realizada neste estudo utilizou bases de dados como o Scopus, BVS, SciELO, PsycInfo, Google Acadêmico e o EBSCO. A presente revisão teve seus dados coletados nas bases separadamente, selecionadas por serem consideradas bases de dados virtuais de referência para publicações nacionais e internacionais em Psicologia.

Os passos de inclusão ou exclusão de artigos para a construção desta pesquisa foram:

1) Utilização do descritor/termo schadenfreude como filtro principal de inclusão de trabalhos científicos, e este deveria aparecer no título ou nas palavras-chaves.

2) Os documentos publicados deveriam ser pertencentes à área da Psicologia. Esse critério foi adotado para excluir estudos que apenas mencionavam o descritor schadenfreude e que foram realizadas em outros campos do conhecimento. Desse modo, fez-se levantamento apenas dos estudos referentes à área da Psicologia que apresentavam como foco principal o estudo da schadenfreude.

3) Selecionados todos os estudos que estivessem publicados em periódicos, revistas especializadas e indexados nas bases de dados mencionadas, a partir do ano de 2009, preservando a importância de estudos atuais, realizados na última década.

4) Foram excluídos documentos que estivessem duplicados entre as bases, cujo tema não considerasse a finalidade proposta neste estudo, ou que não estivessem disponíveis nas plataformas digitais.

A análise dos dados dos documentos indicados foi realizada através de uma ficha de leitura, que buscou identificar quais os principais resultados dos estudos encontrados e suas limitações tendo em vista ser um tema cercado de dificuldades para seu estudo devido à escassez de material. A ficha de leitura foi utilizada pelos pesquisadores, pois a revisão narrativa ou tradicional, quando comparada à revisão sistemática, apresenta uma temática mais aberta, não exigindo um protocolo rígido para sua confecção, e a busca das fontes não é predeterminada e específica, ressaltando a bibliografia incipiente sobre o assunto.

\section{Resultados e Discussão}

Após a busca realizada, foram identificados, inicialmente, 34 documentos, dos quais 17 foram excluídos por não estarem dentre os critérios de inclusão estabelecidos. Dessa forma, a revisão final contemplou um total de 17 documentos, sendo 
16 artigos e 1 dissertação de mestrado. A Tabela 1 apresenta as publicações sobre o tema schadenfreude de acordo com os critérios de inclusão e exclusão adotados nesta revisão narrativa.

Os estudos, em geral, indicam que pessoas que se beneficiaram de alguma forma com uma situação social tendem a expressar a schadenfreude para com os membros do grupo opositor (exogrupo). Além disso, quanto maior o sentimento de identificação com o seu grupo (endogrupo), maior será a possibilidade de expressá-la como uma emoção.

Tabela 1

Publicações sobre schadenfreude no período de 2009 a 2018

\begin{tabular}{|c|c|c|}
\hline Ano & Autores e títulos & Base de dados \\
\hline 2009 & $\begin{array}{l}\text { Combs, D. J. Y., Powell, Caitlin A. J., Sxhurtz, D. R., \& Smith, R. H. Politics, schadenfreude, and } \\
\text { ingroup identification: The sometimes-happy thing about a poor economy and death. }\end{array}$ & Google acadêmico \\
\hline 2009 & $\begin{array}{l}\text { Van Dijk, W. W., Ouwerkerk, J. W. \& Goslinga, S. The impact of deservingness on schadenfreude } \\
\text { and sympathy: further evidence. }\end{array}$ & EBSCO \\
\hline 2010 & Litter, J. \& Cross S. Celebrity and schadenfreude. & EBSCO \\
\hline 2011 & $\begin{array}{l}\text { Van Dijk, W. W., van Koningsbruggen, G. M., Ouwerkerk, J. W., \& Wesseling, Y. M. Self-esteem, } \\
\text { self-affirmation, and schadenfreude. }\end{array}$ & Google acadêmico \\
\hline 2011 & $\begin{array}{l}\text { Monteiro, M. C. Humor e prazer perante a alegria e o infortúnio dos outros: identificação empática, } \\
\text { contágio emocional ou prazer malicioso? }\end{array}$ & $\begin{array}{l}\text { Repositório institucional } \\
\text { do ISCTE-IUL }\end{array}$ \\
\hline 2011 & $\begin{array}{l}\text { Van Dijk, W. W., van Koningsbruggen, G. M., Ouwerkerk, J. W., \& Wesseling, Y. M. Towards } \\
\text { understanding pleasure at the misfortunes of others: the impact of self-evaluation threat on } \\
\text { schadenfreude. }\end{array}$ & EBSCO \\
\hline 2012 & $\begin{array}{l}\text { Cikara, M., \& Fiske, S.T. Stereotypes and schadenfreude: Affective and physiological markers of } \\
\text { pleasure at outgroups' misfortunes. }\end{array}$ & EBSCO \\
\hline 2012 & $\begin{array}{l}\text { Van Dijk, W. W., van Koningsbruggen, G. M., Ouwerkerk, J. W., \& Wesseling, Y. M. "So you } \\
\text { wanna be a pop star?": schadenfreude following another's misfortune on tv. }\end{array}$ & EBSCO \\
\hline 2013 & Colyn, L. A. e Gordon, A. K. Schadenfreude as a mate-value-tracking mechanism. & EBSCO \\
\hline 2013 & Cikara, M., Fiske, S. T. Their pain, our pleasure: Stereotype content and schadenfreude. & Google acadêmico \\
\hline 2014 & $\begin{array}{l}\text { Shamay-Tsoory, S.G, Ahronberg-Kirschenbaum, D., \& Bauminger-Zviely, N. There is no joy like } \\
\text { malicious joy: schadenfreude in young children. }\end{array}$ & EBSCO \\
\hline 2015 & $\begin{array}{l}\text { Boecker, L., Likowski, K. U., Pauli, P., \& Weyers, P. The face of schadenfreude: differentiation of } \\
\text { joy and schadenfreude by electromyography. }\end{array}$ & EBSCO \\
\hline 2015 & Spurgin, E. An emotional-freedom defense of schadenfreude. & EBSCO \\
\hline 2015 & $\begin{array}{l}\text { Van Dijk, W. W, Ouwerkerk, J. W., \& Smith, R. H. Schadenfreude as a mate-value-tracking } \\
\text { mechanism: replication and extension of Colyn and Gordon (2013) }\end{array}$ & Google acadêmico \\
\hline 2016 & $\begin{array}{l}\text { Van de Ven, N., Hoogland, C. E., Smith, Richard H., van Dijk, Wilco W., Breugelmans, S. M., \& } \\
\text { Zeelenberg, M. When envy leads to schadenfreude - cognition and emotion. }\end{array}$ & EBSCO \\
\hline 2018 & $\begin{array}{l}\text { Lange, J., Weidman, A. C., \& Crusius, J. The painful duality of envy: evidence for an integrative } \\
\text { theory and a meta-analysis on the relation of envy and schadenfreude. }\end{array}$ & Google acadêmico \\
\hline 2018 & $\begin{array}{l}\text { Ramos-Oliveira, D., \& Oliveira, F. S. Contribuições da Neurociência Social nos estudos da } \\
\text { schadenfreude, cognição social e emoção intergrupal: revisão integrativa. }\end{array}$ & Google acadêmico \\
\hline
\end{tabular}

Ramos-Oliveira e Oliveira (2018), a partir de uma revisão integrativa de literatura, que une áreas como a neurociência social, a cognição social e a schadenfreude nas relações intergrupais, buscaram identificar como a neurociência social poderia contribuir e oferecer novos insights sobre a cognição social, a schadenfreude e o processamento neural das informações relacionadas às interações sociais. Evidenciou-se uma articulação entre a schadenfreude, a cognição social e suas bases neurais, no intuito de interferirem entre si em um contexto social.

Ainda acerca da cognição social, Cikara e Fiske (2012) investigaram se os estereótipos são suficientes para evocar prazer em relação ao status elevado e aos infortúnios que possam acometer alvos competitivos. Ao examinar se status e competitividade são suficientes para provocar schadenfreude quando tais alvos passarem por uma desgraça, os autores demonstraram que o status percebido e a concorrência entre os pares podem determinar quando e quais alvos evocam schadenfreude, assim como que grupos competitivos de alto status estão mais propensos a serem alvos. Descobriu-se que a schadenfreude pode ser atenuada se membros do grupo foram sensibilizados e informados de que o grupo alvo teria características de cooperação. Os pesquisadores encontraram obstáculos associados com a medição das respostas, que eram classificadas como socialmente indesejáveis, bem como alertam uma dificuldade de associação entre inveja e schadenfreude. 
Essas descobertas, segundo Combs et al. (2009), podem ser associadas às teorias e pesquisas sobre inferioridade grupal, a qual denota que a inferioridade de um grupo em comparação com exogrupos pode criar uma sensação de frustração e vergonha, podendo desencadear uma sensação de raiva e hostilidade em relação ao outro grupo. No entanto há a possibilidade de a maioria das experiências de schadenfreude serem exemplos de sentimentos ambivalentes ou mistos, já que é improvável que pessoas que são bem socializadas se sintam totalmente à vontade com o sofrimento de outras pessoas, especialmente quando elas se sentem egoístas com isso.

É difícil ter a certeza de quantos correlatos de schadenfreude são sentimentos reais dos participantes, pois esses relatórios podem ser autocensurados, mesmo inconscientemente. Como sugestão, os autores trazem a possibilidade de se trabalhar com abordagens de ressonância magnética funcional para avaliar as emoções relacionadas, de maneira que não sejam influenciadas pelo inconsciente.

Tendo a inveja como um dos principais sentimentos relacionados com schadenfreude, Van de Ven et al. (2014) investigaram a relação entre inveja e schadenfreude, tendo por objetivo dissolver as inconsistências encontradas em estudos sobre essa relação. Alguns desses estudos apontam que a inveja acarretaria schadenfreude, enquanto outros garantem que não. Os estudos apresentados por esses autores revelam a distinção entre inveja benigna e maliciosa com o intuito de resolver essa contradição.

Com a inveja maliciosa, o indivíduo centra-se no alvo e deseja que ele não tenha a vantagem que aquele deseja. Na inveja benigna, há um maior centramento no objeto da inveja e de como pode alcançá-lo para si mesmo. Os resultados encontrados apoiaram a ideia de que somente a inveja maliciosa estaria relacionada com mais intensa schadenfreude. Van de Ven et al. (2014) sugerem que pesquisas futuras poderiam testar sentimentos crônicos de inferioridade e o seu efeito sobre a tristeza e também que essas descobertas melhorariam a nossa compreensão sobre os antecedentes de schadenfreude.

Para Lange, Weidman, e Crusius (2018), a schadenfreude e a inveja são correlacionadas positivamente quando a inveja é operacionalizada como maligna, em comparação com quando é operacionalizada pela inveja benigna, ou pela dor. Configura-se uma dúvida, porém, na questão de quando a inveja benigna e a maligna podem evoluir. Pensando nisso, os autores sugerem que as variáveis de merecimento e controle pessoal sejam analisadas como componentes de inveja.

Saindo dos estudos sobre emoções e retomando à associação entre estereótipos e schadenfreude, Cikara e Fiske (2013) ainda revelam que o conteúdo estereotípico relativo ao status e competitividade dos alvos prediz quais grupos são alvos de empatia ou de mal-estar e dano. Além disso, é perceptível que, quando confessar a schadenfreude era socialmente aceitável, o efeito relatado pelo participante afetava os indicadores fisiológicos do prazer e estava associado à disposição de prejudicar os membros dos grupos externos.

Apesar de o conteúdo estereotipado provocar respostas emocionais malignas, esses efeitos são flexíveis, podendo, com a manipulação das percepções de status e competição, haver uma diminuição e eliminação da tristeza em relação aos alvos estereotipadamente invejados. De acordo com os autores (Cikara \& Fiske, 2013), os trabalhos futuros devem interrogar as possíveis funções da schadenfreude no nível intergrupal. Eles trazem justificativas para essa possibilidade, como que a schadenfreude pode funcionar como um sinal de coesão dentro do grupo, já que demonstra prazer, e não empatia, em relação ao infortúnio do outro. É um sinal de que os interesses de alguém não estão alinhados com os da vítima. Além disso, as pessoas podem ser ativamente motivadas a experimentar emoções positivas através do sentimento de schadenfreude, e os grupos podem encontrar maneiras de justificar sua má vontade, promovendo o orgulho em grupo, por exemplo.

Essas ideias de sentimento de inferioridade pessoal ou grupal alinham-se com a pesquisa de Van Dijk, Van Koningsbruggen, Ouwerkerk, e Wesseling (2011a). Eles expõem que pessoas com baixa autoestima experimentam mais schadenfreude do que aqueles que possuem uma autoestima alta, e que essa relação é mediada pela autoameaça. Além disso, descobriu-se que essa relação indireta entre schadenfreude e autoestima depende de uma oportunidade de autoafirmação. Quando nenhuma oportunidade de autoafirmação está disponível, tem-se uma baixa autoestima, experimentando uma autoameaça mais forte.

Esses achados apoiam a suposição de que as conquistas dos outros podem eliciar a schadenfreude, pois fornecem a oportunidade de melhorar ou proteger suas visões pessoais, indicando que a schadenfreude pode representar uma resposta de autoameaça e que a autoafirmação pode atenuá-la por indivíduos com baixa autoestima. As limitações dessa pesquisa estão pautadas no fato de que não foram incluídas condições de confronto com a infelicidade de alcançar o baixo desempenho nas conquistas, não ficando claro se outro alcance é necessário para evocar diferenças na expressão de schadenfreude entre indivíduos de baixa ou alta autoestima (Van Dijk et al., 2011a).

Esses autores apontam que a ameaça ao indivíduo intensifica a schadenfreude, alegando que os infortúnios dos outros podem salientar a preocupação das pessoas por uma visão positiva de si, protegendo, mantendo ou melhorando a autoavaliação desse indivíduo. Quando o infortúnio ocorre em uma área não relacionada à ameaça de autoavaliação, poderá servir como uma função de autoaprimoramento, e também elicia uma escassez de sofrimento perante a situação. Por fim, a pesquisa traz que schadenfreude é uma emoção multideterminada, e enfatiza que pesquisas futuras devem incluir em sua investigação as condições exatas sob as quais os determinantes diferentes e interdependentes evocam a schadenfreude (Van Dijk, Van Koningsbruggen, Ouwerkerk, \& Wesseling, 2011b). 
Van Dijk et al. (2011a) evidenciam, ainda, que os participantes com baixa autoestima eliciam mais schadenfreude depois de terem recebido um feedback negativo diante dos que receberam um feedback positivo. Outro fato explicitado é que uma situação que envolve baixa autoestima e autoameaça induzida provoca um maior prazer diante do infortúnio do outro. Assim, a schadenfreude aparece como satisfação em relação à preocupação de proteger, manter ou aumentar os sentimentos de autoestima.

Os resultados também mostram um efeito de interação entre os participantes, expondo ser mais intensa quando o infortúnio recai sobre uma pessoa que é semelhante à pessoa que está sentindo prazer, servindo como relevante comparação social com o outro, tendo impacto nas relações sociais. Dessa forma, fica a sugestão para pesquisas futuras abordarem schadenfreude e a interação com a simpatia, analisando como iriam afetar as relações sociais.

Já os resultados do estudo de Van Dijk, Ouwerkerk, e Goslinga (2009) mostram que os participantes experimentaram menos schadenfreude em relação a um aluno com conquistas tidas como merecidas do que em relação a um indivíduo com realizações tidas como imerecidas. As análises de mediação mostraram que o efeito da percepção de merecimento de realizações foi totalmente mediado pelo merecimento percebido do infortúnio subsequente. O caminho mediado foi marginalmente significativo para simpatia. Essas descobertas indicam que as reações emocionais para as quedas de grandes empreendedores são mais benignas quando eles percebem essas realizações iniciais como sendo merecidas. Além disso, essas descobertas fornecem mais evidências para o efeito percebido do merecimento da schadenfreude e a simpatia para com pessoas que sofrem infortúnios. As limitações desta pesquisa estão na não apresentação do conceito da schadenfreude, no não aprofundamento do estudo sobre simpatia, infortúnio e schadenfreude, e no fato de não mencionar as consequências do merecimento em schadenfreude e a simpatia para com outras pessoas que sofrem infortúnios.

Tratando de perda da fama de celebridades, Litter e Cross (2010) mostram que a schadenfreude pode ser vista como intimamente ligada a formações econômicas contemporâneas, em virtude de que o fenômeno da celebridade é indicativo de uma sociedade com profunda concentração de poder. Essas demonstrações de expressão de prazer com a perda de prestígio de celebridades referem-se à capacidade de "nivelamento através da humilhação", cumprindo a função cultural específica de oferecer prazer no testemunho do poderoso tornar-se menos poderoso, e uma tentativa de abordar ou lidar com um grave desequilíbrio de poder.

Monteiro (2011) também avalia respostas afetivas diante da alegria e do infortúnio dos outros em situações de humor. Para fazer tal medição, foram coletadas informações da atividade eletrodérmica e aferida medidas de autorrelato para verificar o que os participantes sentiram em cada uma das circunstâncias apresentadas. Parte da amostra mostrou mais prazer com os acidentes cômicos, já outro grupo demonstrou mais prazer com os ataques de riso, e o terceiro sentiu prazer igual diante das duas situações, contudo não se identificou schadenfreude expressiva em nenhum dos grupos em relação aos acidentes. Fora apresentada a sugestão de que, em estudos futuros, uma amostra de maior extensão seja utilizada como participante, pois, assim, outras variáveis poderiam ser contempladas, como o gênero dos voluntários, que foram, em sua maioria, do sexo feminino.

O trabalho de Boecker, Likowski, Pauli, e Weyers (2015) é o primeiro estudo, feito em pessoas do sexo masculino, que teve por objetivo verificar se a schadenfreude, sendo induzida por vídeos, surgiria como resultado do infortúnio alheio e se a emoção apresentada poderia ser distinguida a partir da expressão de alegria utilizando a eletromiografia facial como ferramenta de aferição. Os resultados apresentados trazem similaridades entre alegria e schadenfreude. Foram avaliados estudos de imagem cerebral demonstrando que as duas variáveis emocionais envolvem estruturas cerebrais ligadas aos mecanismos de recompensa, as quais apontariam que a terminologia schadenfreude seria apenas uma construção social, com a finalidade de rotular uma característica moral equivocada de prazer. Contudo, para evidenciar tal argumento, seria necessária uma replicação do estudo para averiguar se eventos de maior repulsa poderiam desencadear expressões mais marcantes de schadenfreude, distinguindo-se da alegria. Além disso, como limitação, a pesquisa deveria ser replicada também com o sexo feminino.

Nesse sentido, pensando na questão de gênero, Colyn e Gordon (2013) indicam que a interação entre gênero e autoameaça teve uma relação significativa com schadenfreude. As análises de acompanhamento mostraram que os participantes do sexo feminino experimentaram, marginalmente, mais essa emoção após o feedback negativo do que após feedback positivo. Os participantes masculinos não apresentaram diferenças em sua experiência de schadenfreude após um ou outro. Os resultados demonstraram também que os participantes com uma baixa autoestima experimentaram mais schadenfreude após ofeedback negativo do que após o feedback positivo. Em contraste, os participantes com uma autoestima alta não diferem em sua experiência de schadenfreude quanto ao feedback.

Van Dijk, Ouwerkerk, e Smith (2015). replicaram e ampliaram esta pesquisa desenvolvida por Colyn e Gordon (2013). Como resultado, os dados sugerem que os participantes do sexo feminino experimentam mais schadenfreude quando outra mulher sofre uma situação de infortúnio do que os homens quando outro homem sofre. Os resultados encontrados foram diferentes dos apresentados por Colyn e Gordon (2013) no que diz respeito à intenção de schadenfreude. No experimento replicado, o infortúnio foi sofrido por alguém conhecido, enquanto na primeira pesquisa o evento negativo aconteceu com um amigo, sugerindo que desgraças sofridas pelos amigos próximos podem restringir expressões de schadenfreude. A 
limitação apontada no estudo é que existiriam inúmeros mecanismos que poderiam eliciar a schadenfreude e é justamente isso que a torna uma emoção intrigante de ser estudada.

Quanto a crianças, os resultados de Shamay-Tsoory, Ahronberg-Kirschenbaum, e Bauminger-Zviely (2014) evidenciam que não demonstram mais schadenfreude para alvos do mesmo sexo em comparação com o alvo do sexo oposto, contradizendo também o modelo de Colyn e Gordon (2013), o que sugere que schadenfreude é um mecanismo psicológico que responde a infortúnios que diminuem o valor social dos concorrentes com a finalidade de aumentar oportunidade de relacionamentos. As análises indicaram que as diferenças apresentadas pelo alvo foram associadas a classificações emocionais mais altas em comparação a igualdades. Além disso, a análise da escala de afetos mostra uma diminuição significativa no afeto positivo, com manipulação de ciúme e uma diminuição significativa no efeito negativo após a manipulação de schadenfreude.

A diferença entre schadenfreude nas classificações ao longo das escalas indica que, de fato, uma situação injusta a provoca. Além disso, aponta que os sinais da expressão da schadenfreude foram observados mesmo nas crianças mais jovens - por volta dos 24 meses de idade.

As hipóteses são de uma origem precoce e evolutiva da aversão à maldade, e indicam que a schadenfreude pode ter evoluído como uma resposta à alocação injusta de recursos. Os dados ainda confirmam se, de fato, as reações emocionais positivas observadas podem ser uma condição para se refletir acerca das reações emocionais associadas à schadenfreude. Os achados estendem a literatura sobre a justiça e a aversão à desigualdade, apresentando o papel de reações emocionais que surgem após condições injustas, ressaltando que os resultados também mostram que as crianças relataram ter sentido a schadenfreude em casa com os pais.

Outro achado interessante que emergiu da análise foi que os índices de ciúme eram mais altos que os de schadenfreude, sugerindo que o ciúme é mais intenso. De fato, tem sido sugerido que o ciúme é mais intenso do que outras emoções baseadas na comparação social, como a inveja, pois talvez envolva um medo extremo de perda de atenção. A pesquisa sobre o ciúme mostra que essas emoções aparecem, mais intensamente, na maioria das crianças, entre, aproximadamente, 13 a 25 meses, e pode ser claramente em torno do terceiro ano de vida. Além disso, existem até relatórios de formas de ciúme em bebês a partir dos 6 meses de idade, indicando que o ciúme é uma emoção poderosa que se desenvolve extremamente cedo na vida.

Por fim, Spurgin (2015) parte de uma defesa da schadenfreude em detrimento daqueles que veem tal emoção como uma falha moral. Para isso, o autor sustenta em seu argumento de que a schadenfreude é permissível em muitos contextos e que ela não seria passível de condenação moral pelo simples fato de ser experimentada e acompanhada variavelmente pelo sentimento de vergonha. $O$ fato de ser uma emoção criticada, e não ser elogiada como positiva, não seria uma razão para não a defender. A alegação principal seria que o sentimento teria um valor instrumental que aparece nos benefícios potenciais em experimentá-la. O estudo prossegue afirmando que os aspectos morais envolvidos nesse processo proporcionam liberdade, no sentido de explorar e compreender o significado do conteúdo dessa emoção, promovendo o crescimento emocional e da saúde mental do indivíduo, contudo acredita-se que ela toma um caráter negativo quando é utilizada para realizar atos imorais.

\section{Considerações Finais}

De um modo geral, este artigo possibilitou um entendimento da perspectiva e compreensão do panorama sobre os estudos da schadenfreude, sendo associada com termos como menos simpatia, merecimento do infortúnio, moralidade, e até mecanismo evolutivo de aversão à iniquidade, os quais estão relacionados às emoções básicas (ex. alegria), emoções mais complexas e emoções competitivas, como a inveja maliciosa e benigna. Além disso, a schadenfreude é associada a ressentimentos, ciúmes e comparação social, sendo compreendida tanto como um sentimento coletivo, como individual de economia afetiva ativados em situação de ameaça de autoavaliação e antipatia em relação ao alvo. Outro fator positivo é a existência de estudos com levantamentos empíricos de coleta de dados que encontraram resultados efetivos, demonstrando que esta é uma área que pode ser mais bem explorada.

Esses achados indicam que a maioria dos artigos correlaciona a schadenfreude não apenas como um mecanismo negativo de prazer ao infortúnio do outro, mas também é correlacionado como um sentimento positivo no âmbito individual, no momento em que satisfaz a preocupação das pessoas por uma visão de si positiva e uma sensação de valor próprio. Sendo assim, correlaciona-se com a inveja benigna, já que é um tipo de força motivacional benéfica, a qual faz com que a pessoa aspire ser tão "boa" quanto a pessoa invejada.

Apesar da importância desses estudos, observa-se que a produção científica nacional em Psicologia ainda é escassa e precisa demonstrar certo crescimento, tanto quanto as produções dos demais países. Uma sugestão para futuros estudos seria a realização, até mesmo replicações, de pesquisas utilizando a realidade brasileira como pano de fundo, tendo em vista a grande parte das produções serem de origem internacional. Tal fato pode contribuir para uma melhor compreensão da schadenfreude atravessada pela realidade brasileira. 


\section{Referências}

Boecker, L., Likowski, K. U., Pauli, P., \& Weyers, P. (2015). The face of schadenfreude: Differentiation of joy and schadenfreude by electromyography. Cognition and Emotion, 29(6), 1117-1125. DOI: 10.1080/02699931.2014.966063

Cikara, M., \& Fiske, S. T. (2012). Stereotypes and schadenfreude: Affective and physiological markers of pleasure at outgroups' misfortunes. Social Psychological Personality Science, 3(1), 63-71. DOI: 10.1177/1948550611409245

Cikara, M., \& Fiske, S. T. (2013). Their pain, our pleasure: Stereotype content and Schadenfreude. Annals of the New York Academy of Sciences, 1299, 52-59. DOI: 10.1111/nyas.12179

Colyn, L. A., \& Gordon. A. K. (2013). Schadenfreude as a mate-value-tracking mechanism. Personal Relationships, 20(3), 524-545. DOI: $10.1111 / \mathrm{j} .1475-6811.2012 .01422 . x$

Combs, D. J. Y, Powell, C. A. J., Sxhurtz, D. R., \& Smith, R. H. (2009). Politics, schadenfreude, and ingroup identification: The sometimes happy thing about a poor economy and death. Journal of Experimental Social Psychology, 45(4), 635646. DOI: $10.1016 /$ j.jesp.2009.02.009

Lange, J., Weidman, A. C., \& Crusius, J. (2018). The painful duality of envy: Evidence for an integrative theory and a metaanalysis on the relation of envy and schadenfreude. Journal of Personality and Social Psychology, 114(4), 572-598. DOI: $10.1037 /$ pspi0000118

Litter.J, \& Cross. S. (2010). Celebrity and schadenfreude.Cultural Studies, 24(3), 395-417.DOI: 10.1080/09502381003750344

Monteiro, M. C. (2011). Humor e prazer perante a alegria e o infortúnio dos outros: Identificação empática, contágio emocional ou prazer malicioso? Dissertação de Mestrado, Departamento de Psicologia Social e das Organizações, ISCTE - IUL, Lisboa, Portugal.

Ramos-Oliveira, D., \& Oliveira, F. S. (2018). Contribuições da neurociência social nos estudos da Schadenfreude, cognição social e emoção intergrupal: Revisão integrativa. Universitas Psychologica, 17(4), 1-12. DOI: 10.11144/Javeriana. upsy17-4.cnse

Rother, E. T. (2007). Revisão sistemática X revisão narrativa. Acta Paulista de Enfermagem, 20(2), 5-6. DOI: 10.1590/ S0103-21002007000200001

Shamay-Tsoory, S. G, Ahronberg-Kirschenbaum, D., \& Bauminger-Zviely, N. (2014). There is no joy like malicious joy: Schadenfreude in young children. PLoSONE 9(7), 100-233. DOI: 10.1371/journal.pone.0100233

Spurgin, E. (2015). An emotional-freedom defense of schadenfreude. Ethical Theory and Modern Practice, 18(4), 767-784. DOI: $10.1007 / \mathrm{s} 10677-014-9550-8$

Van de Ven, N., Hooglandb, C. E., Smithb, R. H., Van Dijkc, W. W., Breugelmansad, S. M., \& Zeelenberga, M. (2014). When envy leads to Schadenfreude. Cognition and Emotion. Advance online publication, 29(6), 1007-1025. DOI: $10.1080 / 02699931.2014 .961903$

Van Dijk, W. W., Ouwerkerk, J. W., \& Goslinga, S. (2009). The impact of deservingness on schadenfreude and sympathy: Further Evidence. The Journal of Social Psychology, 149(3), 290-292. DOI: 10.3200/SOCP.149.3.390-392

Van Dijk, W. W, Ouwerkerk, J. W., \& Smith, R. H. (2015). Schadenfreude as a mate-value-tracking mechanism: Replication and extension of Colyn and Gordon (2013). Personal Relationships, 22(2), 299-307. DOI: 10.1111/pere.12081

Van Dijk, W. W., Van Koningsbruggen, G. M., Ouwerkerk, J. W., \& Wesseling, Y. M. (2011a). Self-esteem, self-affirmation, and schadenfreude. Emotion, 11(6), 1445-1449. DOI: 10.1037/a0026331 
Van Dijk, W. W., Van Koningsbruggen, G. M., Ouwerkerk, J. W., \& Wesseling, Y. M. (2011b). Towards understanding pleasure at the misfortunes of others: The impact of self-evaluation threat on schadenfreude. Cognition and Emotion, 25(2), 360-368. DOI: 10.1080/02699931.2010.487365

Van Dijk, W. W., Van Koningsbruggen, G. M., Ouwerkerk, J. W., \& Wesseling, Y. M. (2012). "So You Wanna Be a Pop Star?": Schadenfreude Following Another's Misfortune on TV. Basic \& Applied Social Psychology, 34 (2), 168-174. DOI: $10.1080 / 01973533.2012 .656006$

\section{Como citar:}

Almeida, S. S. M., Andrade, V., \& Cerqueira, I. (2020). Revisão Narrativa sobre Schadenfreude: O Prazer Malicioso e as Relações Intergrupais. Revista Subjetividades, 20(Esp 1. Relações Intergrupais: Preconceito e Exclusão Social) e8817. http://doi.org/10.5020/23590777.rs.v20iEsp1.e8817

\section{Endereço para correspondência}

Saulo Santos Menezes Almeida

E-mail: saulosma@hotmail.com

Vanessa Andrade de Assis

E-mail: assis@hotmail.com

Ícaro Batista de Cerqueira

E-mail: icaropsi@icloud.com 